\title{
Técnica de distração audiovisual para controlar a ansiedade em crianças no tratamento odontológico.
} Maria Antonia Clara Rodrigues (1) e Carlos Nelson Mendes Rocha (2)

\begin{abstract}
ARTIGO ORIGINAL
Resumo

Objetivo: O objetivo do estudo é avaliar a ansiedade da criança antes e após o tratamento por meio da técnica de distração visual auditiva em crianças.

Metodologia: É um estudo quantitativo, descritivo e transversal. O estudo foi realizado entre 2016 e 2017 em duas clínicas de estomatologia pediátrica. A ansiedade foi avaliada antes e após o tratamento pela técnica audiovisual.
\end{abstract}

Resultados: Verifica-se que a ansiedade leve aumenta após o tratamento de $18 \%$ para $47 \%$. A ansiedade moderada a severa diminui de $16 \%$ para $3 \%$, observa-se que nenhuma criança após o tratamento apresenta ansiedade severa. Esses resultados são estatisticamente significativos.

Conclusões: Em conclusão, a técnica de distração auditiva visual pode ser um método eficaz para reduzir os níveis de ansiedade em pacientes pediátricos durante o tratamento odontológico invasivo.

PALAVRAS CHAVE: Recursos Audiovisuais; Ansiedade; Crianças

Instituição afiliada: 1- Professora da graduação em Odontologia da Faculdade UNImais. 2- Aluno da graduação da Faculdade UNImais.

Dados da publicação: Artigo recebido em 01 de agosto, revisado em 10 de agosto, aceito para publicação em 19 de agosto e publicado em 29 de Agosto.

DOI: https://doi.org/10.36557/2674-8169.2020v2n9p45-53

@ Maria Antonia Clara Rodrigues claraprofodonto@gmail.com 


\section{Audiovisual distraction technique to control anxiety in children in dental treatment.}

Objective: The objective of the study is to assess the child's anxiety before and after treatment using the auditory visual distraction technique in children.

Methodology: It is a quantitative, descriptive and cross-sectional study. The study was carried out between 2016 and 2017 in two pediatric stomatology clinics. Anxiety was assessed before and after treatment using the audiovisual technique.

Results: It appears that mild anxiety increases after treatment from $18 \%$ to $47 \%$. Moderate to severe anxiety decreases from $16 \%$ to $3 \%$, it is observed that no child after treatment has severe anxiety. These results are statistically significant.

Conclusions: In conclusion, the visual auditory distraction technique can be an effective method to reduce anxiety levels in pediatric patients during invasive dental treatment.

KEY WORDS: Audiovisual Resources; Anxiety; Children

Affiliated institution: 1- Professor of Dentistry undergraduate course at Faculdade UNImais. 2-

Undergraduate student at Faculdade UNImais.

Publication data: Article received on August 1, revised on August 10, accepted for publication on August 19 and published on August 29.

DOI: https://doi.org/10.36557/2674-8169.2020v2n9p45-53

@ Maria Antonia Clara Rodrigues claraprofodonto@gmail.com 


\section{INTRODUÇÃO}

O controle da dor, do medo e da ansiedade dos pacientes antes do tratamento odontológico sempre foi uma das partes essenciais da odontologia $\left({ }^{1}\right)$. A ansiedade odontológica é um estado emocional negativo, excessivo e irracional vivenciado pelos pacientes que procuram o consultório odontológico e que ocorre com maior frequência em crianças $\left({ }^{2}\right)$. O que leva a classificá-lo como um problema para a gestão do comportamento infantil com uma prevalência de 3 a $43 \%$ em diferentes populações ( ${ }^{3}$ ) A duração do tratamento odontológico e o manejo do comportamento são dois pontos importantes a serem considerados ao lidar com pacientes infantis. Portanto, tem sido sugerido que as consultas sejam curtas, caso contrário, as crianças interpretariam as sessões de tratamento como um sinal de problemas significativos que causariam ansiedade significativa e, portanto, problemas no gerenciamento do comportamento $\left({ }^{4}\right)\left({ }^{5}\right)$.

Existem diferentes técnicas básicas para diminuir a ansiedade odontológica em crianças durante o tratamento odontológico, como talk show, reforço positivo, comunicação não verbal, controle de voz e distração $\left({ }^{6}\right)\left({ }^{7}\right)$. A distração é uma técnica comum no tratamento odontológico que desvia a atenção da criança do que ela pode perceber como um tratamento desagradável $\left({ }^{8}\right)\left({ }^{9}\right)$ Dentro da técnica de distração existem dois métodos utilizados em odontologia: distração auditiva auditiva e visual. A distração de áudio inclui música, apresentação de áudio e narração de histórias por meio de fones de ouvido. A distração auditiva visual inclui a apresentação de histórias na televisão, realidade virtual e óculos de vídeo 3D. A distração é uma técnica simples que não interfere na comunicação da criança com o dentista $\left({ }^{10}\right)$.

Embora existam diversos estudos sobre essa técnica relacionados ao tratamento odontológico em crianças, estes são controversos, com resultados heterogêneos e têm sido estudados no continente europeu, mas nenhum estudo foi relatado nos continentes americano e africano $\left({ }^{10}\right)$. Nesse sentido, Barreiros $\left({ }^{10}\right)$ recomenda a realização de estudos em outras populações a fim de estabelecer a eficácia da técnica de distração viso-auditiva em crianças de outras origens culturais.

Portanto, o objetivo da presente investigação é avaliar o nível de ansiedade da criança antes e após o tratamento por meio da técnica de distração auditiva visual em crianças.

\section{MATERIAL E MÉTODOS}

É um estudo quantitativo, descritivo e transversal. O estudo foi realizado entre 2016 e 2017 em duas clínicas de estomatologia pediátrica. O estudo teve uma população de 1.161 
crianças, das quais foram selecionadas 400 crianças, 207 do gênero masculino e 193 do gênero feminino, com idades entre 6 e 8 anos.

Foram selecionadas crianças que não sofriam de nenhuma doença sistêmica, e cujo comportamento era levemente negativo, ou seja, aquelas que recusavam o tratamento, faziam movimentos leves com os membros, apresentavam comportamento tímido e, portanto, bloqueio de comunicação, crianças que aceitavam e obedeciam a algumas ordens e cujo grito era monotômico segundo Frankl ( $\left.{ }^{11}\right)$.

A técnica de distração audiovisual ( ${ }^{12}$ ) foi aplicada a todas as crianças, que consistia na apresentação de vídeos narrativos por meio de óculos de realidade virtual. Essa técnica foi realizada durante a execução de tratamentos pulpares (pulpotomias e pulpectomias), sendo classificada como tratamentos invasivos, por implicarem no uso de anestesia infiltrativa.

O nível de ansiedade foi medido com a Escala de Imagem Facial (FIS) ( ${ }^{13}$ ) que tem a ver com o cognitivo (sentimento), onde cada criança era questionada como se sentia antes e depois do tratamento e deveriam responder apontando para o figura com quem ele mais se identificava, então o que foi manifestado pela criança como ansiedade leve, ansiedade leve a moderada, ansiedade moderada, ansiedade moderada a grave e ansiedade severa.

\section{RESULTADOS}

Verifica-se que a ansiedade leve aumenta após o tratamento de $18 \%$ para $47 \%$. A ansiedade moderada a severa diminui de $16 \%$ para 3\%, observa-se que nenhuma criança após o tratamento apresenta ansiedade severa. Esses resultados são estatisticamente significativos.

No sexo masculino, ocorre um aumento da ansiedade de $14 \%$ para $44 \%$. A ansiedade leve a moderada diminuiu de $42 \%$ para $30 \%$. A ansiedade moderada aumentou de $14 \%$ para $24 \%$. A ansiedade moderada a grave diminuiu de $19 \%$ para $2 \%$ e a ansiedade grave diminuiu de $11 \%$ para $0 \%$.

Tabela 1. Nível de ansiedade em crianças antes e após o tratamento

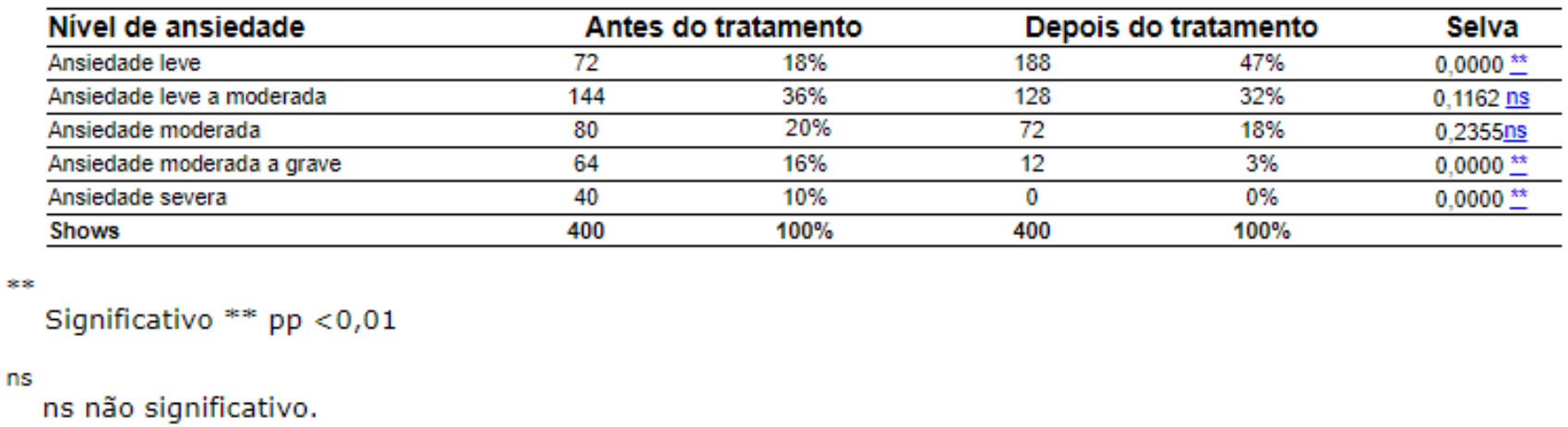


Técnica de distração audiovisual para controlar a ansiedade em crianças no tratamento odontológico.

Maria Antonia Clara Rodrigues e Carlos Nelson Mendes Rocha.

Tabela 2. Nível de ansiedade em crianças antes e após o tratamento de acordo com o sexo

\begin{tabular}{|c|c|c|c|c|c|c|c|c|c|c|}
\hline \multirow{3}{*}{$\begin{array}{l}\text { Nível de ansiedade } \\
\text { Ansiedade leve }\end{array}$} & \multicolumn{5}{|c|}{ MASCULINO } & \multicolumn{5}{|c|}{ FÊMEA } \\
\hline & \multicolumn{2}{|c|}{$\begin{array}{l}\text { Antes do } \\
\text { tratamento }\end{array}$} & \multicolumn{2}{|c|}{$\begin{array}{l}\text { Depois do } \\
\text { tratamento }\end{array}$} & \multirow{2}{*}{\begin{tabular}{|c|}
$\mathbf{P}$ \\
0,0000 \\
$\mathrm{k} \times$ \\
\end{tabular}} & \multicolumn{2}{|c|}{$\begin{array}{c}\text { Antes do } \\
\text { tratamento }\end{array}$} & \multicolumn{2}{|c|}{$\begin{array}{l}\text { Depois do } \\
\text { tratamento }\end{array}$} & \multirow{2}{*}{$\frac{P}{0,0000 \text { 초 }}$} \\
\hline & 29 & $14 \%$ & 92 & $44 \%$ & & $44 \%$ & $2,3 \%$ & $2,3 \%$ & $51 \%$ & \\
\hline Ansiedade leve a moderada & 87 & $42 \%$ & 61 & $30 \%$ & $\underset{\substack{0,0038 \\
\ldots}}{-}$ & 54 & $28 \%$ & $28 \%$ & $35 \%$ & $\begin{array}{c}0,9231 \\
\underline{\underline{\mathrm{nS}}} \\
\end{array}$ \\
\hline Ansiedade moderada & 29 & $14 \%$ & $51^{c}$ & $24 \%$ & 0,0043 & 54 & $28 \%$ & $28 \%$ & $9 \%$ & 0,0000 *t* \\
\hline $\begin{array}{l}\text { Ansiedade moderada a } \\
\text { grave }\end{array}$ & 40 & $19 \%$ & 4 & dois\% & 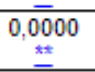 & 22 & $12 \%$ & $12 \%$ & $5 \%$ & $0,0075 \underline{\text { ns }}$ \\
\hline Ansiedade severa & 22 & onze $\%$ & 0 & $0 \%$ & 0,0000 & 18 & $9 \%$ & $9 \%$ & $0 \%$ & $0,0000 \stackrel{\star \star \star}{-}$ \\
\hline Total & 207 & $100 \%$ & 207 & $100 \%$ & & 193 & $100 \%$ & $100 \%$ & $100 \%$ & \\
\hline
\end{tabular}

Significativo $* *$ pp $<0,01$

ns

ns não significativo.

No sexo feminino é apreciado um aumento de $23 \%$ a $51 \%$. A ansiedade moderada diminuiu de $28 \%$ para $9 \%$. A ansiedade moderada a grave diminuiu de $12 \%$ para $5 \%$ e na ansiedade grave houve uma diminuição de $9 \%$ para $0 \%$

\section{DISCUSSÃO}

Ao avaliar o efeito da técnica de distração auditiva visual em crianças com diferentes níveis de ansiedade, evidencia-se que a ansiedade leve aumenta, a ansiedade moderada a severa e a ansiedade severa diminuem significativamente após o tratamento, o que mostra que a técnica de distração auditiva $O$ visual diminui a ansiedade em crianças, conforme demonstrado por diversos estudos que demonstram as atitudes positivas de pacientes pediátricos $\left({ }^{10}\right)\left({ }^{14}\right)$.

Além disso, há estudos como os realizados por Al - Khotani ( ${ }^{15}$ ) e Prabhakar $\left({ }^{16}\right)$ em que diferentes escalas foram adicionadas para avaliar o nível de ansiedade, como o exame de imagem de Venham, a classificação de ansiedade clínica de Venham, comportamento cooperativo (MVARS), frequência de pulso e saturação de oxigênio, além da Escala de Imagem Facial (FIS), apresentando resultados semelhantes ao presente estudo. A estes se acrescenta o realizado por Mitrakul ( ${ }^{21}$ ) que avaliou a ansiedade nas diferentes etapas do procedimento odontológico.

No entanto, Ram $\left({ }^{17}\right)$ não encontrou uma diminuição considerável na ansiedade usando esta técnica, mas ele descobriu que, quando comparada com o óxido nitroso, ambas as técnicas tinham eficácia semelhante. A inalação de óxido nitroso / oxigênio demonstrou diminuir a ansiedade $\left({ }^{18}\right)$, razão pela qual os resultados obtidos neste estudo podem ser explicados. Agarwal ( ${ }^{22}$ ) comparou a eficácia de dois anestésicos tópicos no controle da dor 
e da ansiedade em crianças durante a aplicação de anestesia odontológica, utilizando auxílio audiovisual, obtendo resultados semelhantes ao presente estudo.

A duração e a complexidade do tratamento odontológico são fatores que influenciam diretamente no nível de ansiedade em pacientes pediátricos; Para o presente estudo, foram realizados tratamentos invasivos e (pulpotomias e pulpectomias), nos quais houve ligeiro aumento da ansiedade nos pacientes, como os encontrados nos estudos de Aminabadi ( ${ }^{4}$ ) e Jamali $\left({ }^{5}\right)$. Outro estudo, como o realizado por Hanif $\left({ }^{20}\right)$, indica que, além dos fatores citados, o nível socioeconômico e as consultas odontológicas anteriores influenciam o comportamento e a ansiedade desses pacientes.

Para fazer parte do estudo, foram selecionadas crianças entre 6 e 8 anos, por apresentarem geralmente um comportamento classificado segundo Frankl como levemente negativo e de difícil controle. A isso, acrescenta-se que nesta idade as crianças já estão maduras o suficiente para interagir com a técnica de distração viso-auditiva durante procedimentos odontológicos. Aminabadi $\left({ }^{19}\right)$, argumenta que as técnicas de gestão do comportamento devem ser aplicadas dependendo da idade, características cognitivas e padrões de comportamento.

Pelo fato de existirem poucos estudos semelhantes realizados na América Latina, estamos diante de um estudo inovador que fornecerá subsídios importantes para futuras pesquisas na área de Odontopediatria na região para melhorar a qualidade do atendimento aos pacientes ansiosos.

\section{CONCLUSÕES}

Em conclusão, a técnica de distração auditiva visual pode ser um método eficaz para reduzir os níveis de ansiedade em pacientes pediátricos durante o tratamento odontológico invasivo.

\section{REFERÊNCIAS}

1. Angelo Z, Polyvios C. Alternative practices of achieving anaesthesia for dental procedures: a review. J Dent Anesth Pain Med. abril de 2018;18(2):79-88.

2. Soares Fernandes, A. P., \& Battistella, M. A. Dental Implants in Pediatric Dentistry: A Literature Review. Brazilian Journal of Implantology and Health Sciences. (2020); 2(2):1-12. 
3. Lee C-Y, Chang Y-Y, Huang S-T. Prevalence of dental anxiety among 5- to 8-year-old Taiwanese children. J Public Health Dent. 2007;67(1):36-41

4. Aminabadi NA, Oskouei SG, Farahani RMZ. Dental treatment duration as an indicator of the behavior of 3-to 9-year-old pediatric patients in clinical dental settings. J Contemp Dent Pract. 1 de septiembre de 2009;10(5):E025-032

5. Jamali Z, Najafpour E, Ebrahim Adhami Z, Sighari Deljavan A, Aminabadi NA, Shirazi S. Does the length of dental procedure influence children's behavior during and after treatment?A systematic review and critical appraisal. J Dent Res Dent Clin Dent Prospects. 2018;12(1):68-76.

6. Adair SM, Rockman RA, Schafer TE, Waller JL. Survey of behavior management teaching in pediatric dentistry advanced education programs. Pediatr Dent. abril de 2004;26(2):151-8.

7. Buchanan H, Niven N. Self-report treatment techniques used by dentists to treat dentally anxious children: a preliminary investigation. Int J Paediatr Dent. enero de 2003;13(1):9-12.

8. Clinical Affairs Committee-Behavior Management Subcommittee, American Academy of Pediatric Dentistry. Guideline on Behavior Guidance for the Pediatric Dental Patient. Pediatr Dent. octubre de 2015;37(5):57-70.

9. Brignardello-Petersen R. Audiovisual distraction resulted in less operator stress than behavior management techniques in cooperative children with special health care needs at the dental office, but there was no improvement in behavior, pain, or appointment time. J Am Dent Assoc. 1 de octubre de 2017;148(10):e138.

10. Barreiros D, Oliveira DSB de, Queiroz AM de, Silva RAB da, Paula-Silva FWG de, Küchler EC. Audiovisual distraction methods for anxiety in children during dental treatment: $A$ systematic review and meta-analysis. J Indian Soc Pedod Prev Dent. 1 de enero de 2018;36(1):2.

11. al TA et. A Study of the Relationship of Parenting Styles, Child Temperament, and Operatory Behavior in Healthy Children. - PubMed - NCBI [Internet].

12. Blumer S, Khoury RS, Peretz B. The Prevalence of ADHD Patients among Pediatric Dentists in Israel and Knowledge of Dental and Behavioral Aspects of Treating Them. J Clin Pediatr Dent. 2018;42(3):212-6.

13. Buchanan $\mathrm{H}$, Niven N. Validation of a Facial Image Scale to assess child dental anxiety. Int J Paediatr Dent. enero de 2002;12(1):47-52.

14. Zhang C, Qin D, Shen L, Ji P, Wang J. Does audiovisual distraction reduce dental anxiety in children under local anesthesia?A systematic review and meta-analysis. Oral Dis. 2 de marzo de 2018; 
15. Al-Khotani A, Bello LA, Christidis N. Effects of audiovisual distraction on children's behaviour during dental treatment:a randomized controlled clinical trial. Acta Odontol Scand. 17 de agosto de 2016;74(6):494-501.

16. Prabhakar AR, Marwah N, Raju OS. A comparison between audio and audiovisual distraction techniques in managing anxious pediatric dental patients. J Indian Soc Pedod Prev Dent. 10 de enero de 2007;25(4):177

17. Ram D, Shapira J, Holan G, Magora F, Cohen S, Davidovich E. Audiovisual video eyeglass distraction during dental treatment in children. Quintessence Int Berl Ger 1985. septiembre de 2010;41(8):673-9.

18. Guideline on Use of Nitrous Oxide for Pediatric Dental Patients. Pediatr Dent. octubre de 2016;38(6):211-5.

19. Asl Aminabadi N, Erfanparast L, Sohrabi A, Ghertasi Oskouei S, Naghili A. The Impact of Virtual Reality Distraction on Pain and Anxiety during Dental Treatment in 4-6 Year-Old Children:a Randomized Controlled Clinical Trial. J Dent Res Dent Clin Dent Prospects. 2012;6(4):117-24.

20. Hanif Raja G, Shafiq Malik F, Bashir U. Dental Anxiety among children of age between 5 to 10 years visiting a teaching dental hospital in Islamabad. Pakistan. J Ayub Med Coll Abbottabad 2015;27(3):587-90.

21. Mitrakul K, Asvanund Y, Arunakul M, Paka-Akekaphat S. Effect of audiovisual eyeglasses during dental treatment in 5-8 year-old children. Eur J Paediatr Dent. 2015;16 (3):239-45.

22. Gomes Machado, C. P. ., A. . Vaz Braga Pintor, e M. D. . Calasans Maia. "Evaluation of Strontium-Containing Hydroxyapatite As Bone Substitute in Sheep Tibiae.". Brazilian Journal of Implantology and Health Sciences, vol. 1, no 7, dezembro de 2019, p. 153-64, doi:10.36557/2674-8169.2019v1n7p153-164. 